\title{
A Review on Microstrip Antennas Integrated with EBG Structure
}

\author{
Mrs. Kirti Varma ${ }^{1}$, Prof. Atmeshkumar Patel ${ }^{2}$ \\ ME Student, Department of E\&TC, MSSCET, Jalna, Maharashtra, India ${ }^{1}$ \\ Asst. Professor, Department of E\&TC, MSSCET, Jalna, Maharashtra, India ${ }^{2}$
}

\begin{abstract}
This paper contains, developing and employing methods to reduce the mutual coupling between elements of an antenna array. The utilization of electromagnetic band-gap (EBG) structures is an attractive way to reduce surface wave excitation in printed antenna geometries so to mitigate the mutual coupling problem. This paper investigates the performance of a microstrip antenna phased array embedded in an EBG. The band-gap feature of surface-wave suppression is demonstrated by plotting variations of the transmission coefficient S12 with frequency and dispersion diagram. The antenna design is verified by High Frequency Structural Simulator (HFSS), the simulating results show that the EBG design approach is a good candidate for a reduction in mutual coupling at certain frequencies between radiator elements, which in turn increases antenna directivity. Its band-gap feature of surface-wave suppression is demonstrated by exhibiting the near field distributions of the electromagnetic waves. The mutual coupling of microstrip antennas is parametrically investigated, including the $\mathrm{E}$ and $\mathrm{H}$ coupling directions, different substrate thickness, and various dielectric constants. It is observed that the E-plane coupled microstrip antenna array on a thick and high permittivity substrate has a strong mutual coupling due to the pronounced surface waves. Therefore, an EBG structure is inserted between array elements to reduce the mutual coupling.
\end{abstract}

Keywords: Electromagnetic band-gap (EBG), High Frequency Structural Simulator (HFSS), microstrip antennas, mutual coupling, surface wave.

\section{INTRODUCTION}

Electromagnetic band gaps (EBGs) with their periodic-like structures has gained significant interest in academia and RF-microwave industries due to their extraordinary surface wave suppression property. The band -gap features of an EBG are revealed in two ways: the suppression of surface-wave propagation travels along the structure in specified frequency bands and is guided in a direction that is desirable by forbidding the propagation of EM waves into certain frequency bands, and the in-phase reflection coefficient. Therefore, if the EBG structure is applied to the mobile phone antennas, the undesired electromagnetic waves can be prevented, and the band frequency can be notched. Surface-wave suppression helps to improve an antenna's performance by increasing the antenna gain and reducing back radiation. Various EBG structures have been studied extensively in the last decade. Linear array of microstrip antennas are popular candidates for multiple input multiple output (MIMO) systems and radar applications such as spatial tracking. An important requirement for these applications is high integration densities to utilize available substrate space efficiently. The electromagnetic band-gap (EBG) structure in the electromagnetic and antenna community is based on the photonic band-gap (PBG) phenomena in optics that are realized by periodical structures. This paper focuses on a mushroom-like EBG structure, as shown in Fig. 1. Compared to other EBG structures such as dielectric rods and holes, this structure has a winning feature of compactness, which is important in communication antenna applications. Its band-gap features are revealed in two ways: the suppression of surface-wave propagation, and the in-phase reflection coefficient. The feature of surface-wave suppression helps to improve antenna's performance such as increasing the antenna gain and reducing back radiation. Meanwhile, the in-phase reflection feature leads to low profile antenna designs.

This paper concentrates on the surface-wave suppression effect of the EBG structure and its application to reduce the mutual coupling of microstrip antennas, as shown in Fig. 1. To explore the surface-wave suppression effect, the propagating fields of an infinitesimal dipole source with and without the EBG structure are simulated and compared using the finite difference time-domain (FDTD) method, and a frequency stopband for the field propagation is identified.

Applications of microstrip antennas on high dielectric constant substrates are of special interest due to their compact size and conformability with the monolithic microwave integrated circuit (MMIC). However, the utilization of a high dielectric constant substrate has some drawbacks. Among these are narrower bandwidths and pronounced surface waves. The bandwidth can be recovered using a thick substrate, yet this excites severe surface waves. The generation of 
surface waves decreases the antenna efficiency and degrades the antenna pattern. Furthermore, it increases the mutual coupling of the antenna array which causes the blind angle of a scanning array.

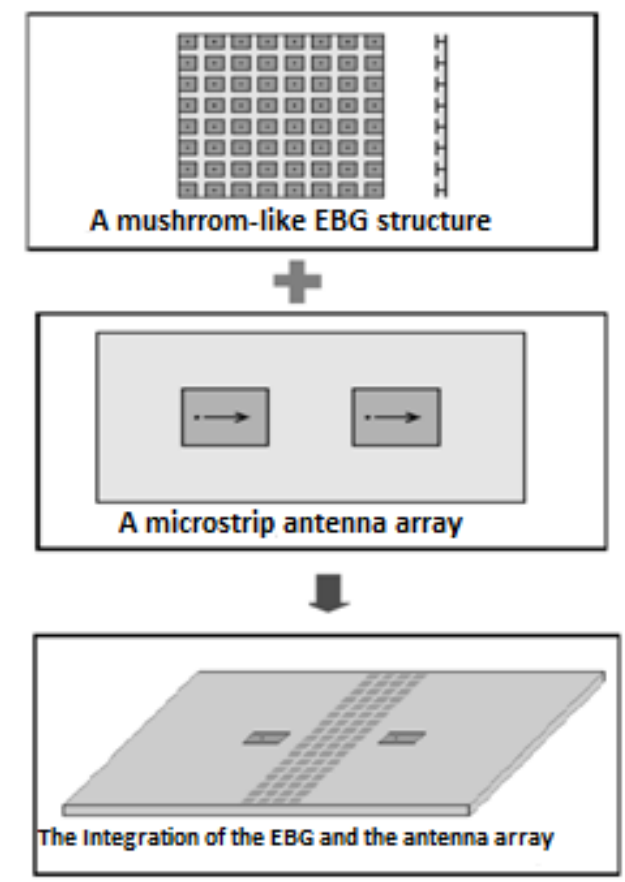

Fig.1. Integration of the EBG structure with MSA array

\section{LITERATURE SURVEY}

Fan Yang and Yahya Rahmat-Samii proved that the Utilization of electromagnetic band-gap (EBG) structures is becoming attractive in the electromagnetic and antenna community. A mushroom-like EBG structure is analyzed using the finite-difference time-domain (FDTD) method. Its band-gap feature of surface-wave suppression is demonstrated by exhibiting the near field distributions of the electromagnetic waves. The mutual coupling of microstrip antennas is parametrically investigated, including the $\mathrm{E}$ and $\mathrm{H}$ coupling directions, different substrate thickness, and various dielectric constants. It is observed that the E-plane coupled microstrip antenna array on a thick and high permittivity substrate has a strong mutual coupling due to the pronounced surface waves. Therefore, an EBG structure is inserted between array elements to reduce the mutual coupling. This idea has been verified by both the FDTD simulations and experimental results. As a result, a significant $8 \mathrm{~dB}$ mutual coupling reduction is noticed from the measurements [1].

Y Rahmat-Samii and H Mosallaei describes that the Periodic structures are abundant in nature. The characteristics such as frequency stop-bands, pass-bands and band-gaps could be identified. They observe that various terminology have been used depending on the domain of the applications. These applications are seen in filter designs, gratings, frequency selective surfaces (FSS), photonic crystals and band-gaps (PBG), etc. We classify them under the broad terminology of "Electromagnetic Band-gaps (EBG)". The focus of this paper is to present a powerful computational engine utilizing Finite Difference Time Domain (FDTD) technique integrated with the Prony method to analyze and understand the unique propagation characteristics of different classes of complex EBG structures such as, (a) FSS structures, (b) PBG crystals, (c) smart surfaces for communication antenna applications, (d) surfaces with perfectly magnetic conducting properties (PMC), (e) creation of materials with negative permittivity and negative permeability, ( f) surfaces with reduced edge diffraction effects, and (g) the notion of equivalent media. The performance of two types of the EBG structures namely, single and multi-layered tripod FSS, and rectangular, triangular and woodpile PBG crystals is detailed [2].

Alon S. Barlevy and Yahya Rahmat-Samii describes the performance of an electromagnetic band-gap structure. The structure is purely metallic and infinitely periodic in two dimensions with a finite periodicity in the third dimension. An effective band-gap exists when within a certain frequency range, the reflection is 100\% for all angles of incidence and all polarizations. The performance of the structure is explained from a physical point of view by isolating the effects of each parameter of the structure. Cascading two periodic arrays with a very small separation distance gives a capacitance effect for the overlap region. The capacitance is shown to control the lower edge of the band-gap. Cascading more closely coupled periodic arrays gives a rejection band, with the separation controlling the upper edge of the band. An effective band-gap is shown to exist when different layers are connected with vias. The structure examined in this paper has an effective band-gap from $30 \mathrm{GHz}$ to $100 \mathrm{GHz}$ [3]. 
Ramon Gonzalo, Peter de Maagt, Mario Sorolla explained the microstrip patch antenna is a low-profile robust planar structure. A wide range of radiation patterns can be achieved with this type of antenna and, due to the ease of manufacture, is inexpensive compared with other types of antennas. However, patch-antenna designs have some limitations such as restricted bandwidth of operation, low gain, and a potential decrease in radiation efficiency due to surface-wave losses. In this paper, a photonic-bandgap (PBG) substrate for patch antennas is proposed, which minimizes the surface-wave effects. In order to verify the performance of this kind of substrate, a configuration with a thick substrate is analyzed. The PBG patch antenna shows significantly reduced levels of surface modes compared to conventional patch antennas, thus improving the gain and far-field radiation pattern [4].

Li Yang, Zhenghe Feng, Fanglu Chen, and Mingyan Fan describe a novel electromagnetic band-gap (EBG) structure in a fork-like shape. The period of the EBG lattice is only O.W\& at the desired stopband frequency, resulting in the most compact EBG lattice ever achieved A comparison has been carried out between the new structure and the mushroom-like EBG structure. Simulations and experimental results have verified that the area of the fork like structure is less than $40 \%$ of the latter. Also a double element microstrip antenna array inserted with the new EBG structure has been simulated, showing that a $6.51 \mathrm{~dB}$ mutual coupling reduction is achieved [5].

\section{II.MUTUAL COUPLING COMPARISON OF VARIIOUS MICROSTRIP ANTENNA ARRAYS}

\section{A. FDTD Method for Mutual Coupling Simulation:}

The FDTD method is used to analyze the mutual coupling of microstrip antennas. The mutual coupling of antennas fed by microstrip lines has been solved using the FDTD method and the probe fed antenna case is discussed herein. Fig. 5 plots an FDTD model to calculate the mutual coupling of two probe fed patch antennas. The reflection coefficients are defined as

$$
\left[\begin{array}{l}
b 1 \\
b 2
\end{array}\right]=\left[\begin{array}{ll}
511 & 512 \\
\$ 21 & \$ 22
\end{array}\right]\left[\begin{array}{l}
a 1 \\
a 2
\end{array}\right]
$$

Where, a1, a2, b1 and b2 are the normalized voltage waves. The incident wave and reflected waves are mixed together during the FDTD simulation, and the voltages and currents are recorded at the ports.

A Gaussian pulse type of voltage source is used to excite the structure. For simplicity, only port one is activated during the simulation and port two is matched to 50. Therefore, the incident wave at port two is zero;

$$
\begin{aligned}
& b_{1}=S_{11} a_{1} \\
& b_{2}=S_{21} a_{1}
\end{aligned}
$$

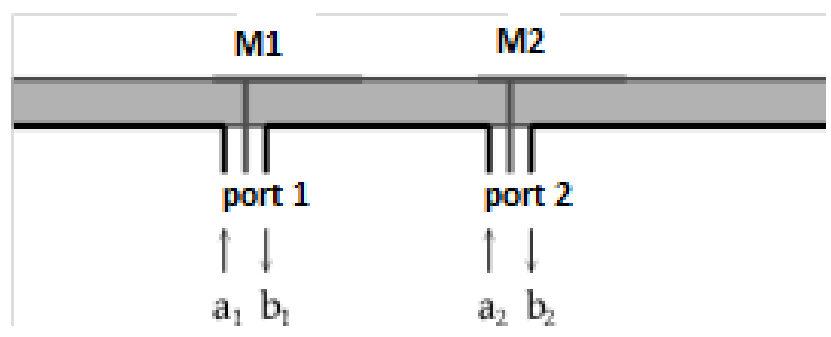

Fig.2. FDTD model to calculate the mutual coupling of probe fed MSA

B. Mutual Coupling Comparison:

The developed FDTD method is next used to analyze the mutual coupling features of microstrip antennas at different thicknesses and permittivity. Both the E-plane and H-plane couplings are investigated, and four patch antennas are compared as follows:

1) Patch antennas on a thin and low dielectric constant substrate: $€_{r}=2.20, h=1 \mathrm{~mm}$, and the patch size is $16 \mathrm{~mm} X 9$ $\mathrm{mm}$;

2) Patch antennas on a thick and low dielectric constant substrate: $€_{\mathrm{r}}=2.20, \mathrm{~h}=2 \mathrm{~mm}$, and the patch size is $15.5 \mathrm{~mm} X$ $12 \mathrm{~mm}$;

3) Patch antennas on a thin and high dielectric constant substrate: $€_{\mathrm{r}}=10.20, \mathrm{~h}=1 \mathrm{~mm}$, and the patch size is $7.5 \mathrm{~mm} X 5$ $\mathrm{mm}$;

4) Patch antennas on a thick and high dielectric constant substrate: $€_{\mathrm{r}}=10.20, \mathrm{~h}=2 \mathrm{~mm}$, and the patch size is $7 \mathrm{~mm} X$ $\mathrm{mm}$.

It can be observed that the bandwidth increases with increasing thickness and decreases while the permittivity increases. It's worthwhile to point out that the bandwidth of case 4 is even larger than that of case 1 , which means the bandwidth of microstrip antennas on a high permittivity substrate can be recovered by increasing the substrate 
thickness. In contrast to the E-plane coupled results, the strongest mutual coupling occurs at the second case, which has a low dielectric constant and a thick substrate thickness, and the weakest mutual coupling happens at the third case, which has a high dielectric constant and a thin substrate thickness. It is observed that increasing the substrate thickness still increases the mutual coupling, while increasing the permittivity decreases it.

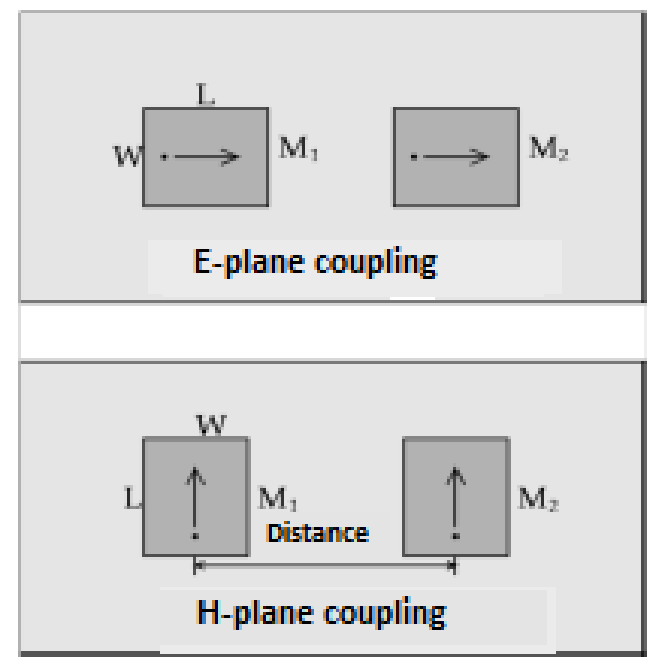

Fig.3. E and H-plane coupled probe fed microstrip antennas.

\section{ANTENNA PARAMETERS}

Some basic antenna parameters such as Antenna Gain, VSWR, Radiation Pattern, Radiation Intensity, Return Loss, Beamwidth, Directivity, Effective Aperture, Antenna Efficiency, Bandwidth and Input Impedance etc. are studied.

Antenna Gain $(\mathrm{G})$ :

The gain of an antenna is defined as the ratio of the intensity, in a given direction, to the radiation intensity that would be obtained if the power accepted by the antenna were radiated isotropically.

$$
\mathrm{G}=4 \pi * \mathrm{U}(\theta, \Phi) / \text { Pin }
$$

Where, $\mathrm{U}(\theta, \Phi)$ is radiation intensity in a given direction,

Pin is total input power.

Voltage standing wave ratio (VSWR):

Voltage standing wave ratio is defined as

$\mathrm{VSWR}=\mathrm{Vmax} / \mathrm{Vmin}$.

It should lie between 1 and 2 .

2. Beamwidth:

The beamwidth of a power pattern is defined as the angular separation between two identical points on opposite side of the pattern maximum.

\section{Radiation Pattern:}

The radiation pattern is defined as a mathematical function or a graphical representation of the radiation properties of the antenna as a function of space coordinates. The Pattern is three dimensional figure plotted on (x, y, z) axis, in which we have major lobe, minor lobe, side lobe, back lobe, first null beamwidth (FNBW), half power beamwidth (HPBW).

\section{CONCLUSION}

In this paper, the survey of likely research papers are completed to cover an area of mushroom EBG structure for array miniaturization with reduced mutual coupling. EBG structure is analyzed using the An soft HFSS. EBG structures for a several operating frequencies have been presented and several useful properties of the structure have been investigated, like the in-phase band gap reflection coefficient and S-parameters. These properties make the proposed EBG structure a good candidate to enable the design of flow profile antennas, and the surface wave suppression band gap property which improves the antenna performance. 


\section{IJARCCE}

Vol. 6, Issue 6, June 2017

\section{REFERENCES}

[1] Fan Yangand Yahya Rahmat-Samii, Member IEEE "Microstrip Antennas Integrated with Electromagnetic Band-Gap (EBG) Structures: A Low Mutual Coupling Design for Array Applications" IEEE transaction on antennas and propagation, vol. 51, no. 10, October 2003.

[2] Y Rahmat Samii And H Mosallaei "Electromagnetic Band-Gap Structures:Classification, Characterization, and Applications" 17th International Conference On Antennas And Propagation, 17-20 April 2001, Conference Publication No. 480 IEEE 2001.

[3] Alon S. Barlevy and Yahya Rahmat-Samii "High-Impedance Electromagnetic Surfaces with AForbidden Frequency Band" IEEE Transactions On Microwave Theory And Techniques, Vol. 47, No. 11, November 1999.

[4] R. Gonzalo, P. Maagt, and M. Sorolla, "Enhanced patch-antenna performance by suppressing surface waves using photonic-bandgap substrates," IEEE Trans. Microwave Theory Tech., vol. 47, pp. 2131-2138, Nov. 1999.

[5] Li Yang, Zhenghe Feng, Fanglu Chen, and Mingyan Fan, "Electromagnetic Band Gap Structures In-Corporate With Dual Band Microstrip Antenna Array" Progress In Electromagnetics Research M, Vol. 11, 111, 2010.

[6] ShahramMohanna, Ali Farahbakhsh, And SaeedTavakoli, "Mutual Coupling Reduction In Two-Dimensional Array Of Microstrip AntennasUsing Concave Rectangular Patches" Journal Of Telecommunications, Volume 2, Issue 2, May 2010.

[7] HamidehKondori, Mohammad Ali Mansouri-Birjandi "Reducing Mutual Coupling In Microstrip ArrayAntenna Using Metamaterial Spiral Resonator" Ijcsi International Journal Of Computer Science Issues, Vol. 9, Issue 3, No 1, May 2012.

[8] Abdelmoumen Kaabal, Mustapha El halaoui "A LowMutual Coupling Design for Array MicrostripAntennasIntegrated with Electromagnetic Band-Gap Structures" 9th International Conference Interdisciplinarity in Engineering, INTER-ENG 2015, 8-9 October2015.

[9] M. Rahman and M. Stuchly, "Wide-band microstrip patch antenna with planar PBG structure," in Proc. IEEE AP-S Dig., vol. 2, July 2001, pp. 486-489.

[10] R. Coccioli, F. R. Yang, K. P. Ma, and T. Itoh, “Aperture-coupled patch antenna on UC-PBG substrate," IEEE Trans. Microwave Theory Tech., vol. 47, pp. 2123-2130, Nov. 1999 